\title{
Determinants of infant breastfeeding practices in Nepal: a national study
}

\author{
Shiva Bhandari ${ }^{1,2^{*}}$, Andrew L. Thorne-Lyman ${ }^{3}$, Binod Shrestha ${ }^{1}$, Sumanta Neupane ${ }^{1}$, \\ Bareng Aletta Sanny Nonyane ${ }^{3}$, Swetha Manohar ${ }^{3}$, Rolf D. W. Klemm, and Keith P. West $\mathrm{Jr}^{3}$
}

\begin{abstract}
Background: Optimal breastfeeding practices, reflected by early initiation and feeding of colostrum, avoidance of prelacteal feeds, and continued exclusivity or predominance of breastfeeding, are critical for assuring proper infant nutrition, growth and development.

Methods: We used data from a nationally representative survey in 21 district sites across the Mountains, Hills and Terai (southern plains) of Nepal in 2013. Determinants of early initiation of breastfeeding, feeding of colostrum, prelacteal feeding and predominant breastfeeding were explored in 1015 infants $<12$ months of age. Prelacteal feeds were defined as food/drink other than breast milk given to newborns in first 3 days. Predominant breastfeeding was defined as a child $<6$ months of age is mainly breastfed, not fed solid/semi-solid foods, infant formula or non-human milk, in the past 7 days. Adjusted prevalence ratios (APR) and 95\% confidence intervals (Cl) were estimated, using log Poisson regression models with robust variance for clustering.
\end{abstract}

Results: The prevalence of breastfeeding within an hour of birth, colostrum feeding, prelacteal feeding and predominant breastfeeding was 41.8, 83.5, 32.7 and 57.2\% respectively. Compared to infants not fed prelacteal feeds, infants given prelacteal feeds were $51 \%$ less likely to be breastfed within the first hour of birth (APR $0.49 ; 95 \% \mathrm{Cl} 0.36$, 0.66 ) and $55 \%$ less likely to be predominantly breastfed (APR 0.45; $95 \% \mathrm{Cl} 0.32,0.62$ ). Infants reported to have received colostrum were more likely to have begun breastfeeding within an hour of birth (APR 1.26; 95\% Cl 1.04, 1.54) compared to those who did not receive colostrum. Infants born to mothers $\geq 20$ years of age were less likely than adolescent mothers to initiate breastfeeding within 1 hour of birth. Infants in the Terai were $10 \%$ less likely to have received colostrum (APR 0.90; 95\% Cl 0.83, 0.97) and 2.72 times more likely to have received prelacteal feeds (APR 2.72; 95\% Cl 1. $67,4.45)$ than those in the Mountains.

Conclusions: Most infants in Nepal receive colostrum but less than half initiate breastfeeding within an hour of birth and one-third are fed prelacteal feeds, which may negatively affect breastfeeding and health throughout early infancy.

Keywords: Breastfeeding, Colostrum, Infant, Prelacteal feeding, Nepal

\section{Background}

Appropriate and optimal infant feeding is fundamentally important to assure adequate nutrition and growth during infancy. Optimal breastfeeding involves complementary feeding and overlapping practices of exclusive breastfeeding (breastmilk with no other foods or liquids) for the first 6 months of life, early inititiation of breastfeeding as soon as a child is born, feeding colostrum and

\footnotetext{
* Correspondence: bhandarishv@gmail.com

${ }^{1}$ PoSHAN Study Team, Lalitpur, Nepal

${ }^{2}$ Department of Health Promotion, Education and Behavior, Arnold School of

Public Health, University of South Carolina, Columbia, SC, USA

Full list of author information is available at the end of the article
}

avoiding prelacteal foods [1]. In Nepal and elsewhere throughout South Asia, suboptimal infant feeding practices have been associated with undernutrition, reflected by stunting and wasting, and mortality [2-4]. Practices such as early initiation of breastfeeding, avoiding prelacteal feeds, assuring intake of colostrum and maintaining exclusivity of breastfeeding in early infancy, represent critical exposures that benefit child growth and development $[5,6]$. Exclusive breastfeeding up to 6 months of age and continuance of breastfeeding during the first [7] and second [8] years of life have been associated with

(c) The Author(s). 2019 Open Access This article is distributed under the terms of the Creative Commons Attribution 4.0 International License (http://creativecommons.org/licenses/by/4.0/), which permits unrestricted use, distribution, and reproduction in any medium, provided you give appropriate credit to the original author(s) and the source, provide a link to the Creative Commons license, and indicate if changes were made. The Creative Commons Public Domain Dedication waiver (http://creativecommons.org/publicdomain/zero/1.0/) applies to the data made available in this article, unless otherwise stated. 
increased linear growth and better cognitive development scores [9].

The World Health Organization (WHO) recommends that mothers practice exclusive breastfeeding for the first 6 months of life, followed by a timely introduction of appropriate complementary foods [10]. Early initiation of breastfeeding (i.e. within $1 \mathrm{~h}$ of birth) is recommended as the first critical step to ensure children receive colostrum, the "first milk" which is rich in nutrients and antibodies essential for rapid adaptation to postnatal life. Early suckling can also facilitate success with subsequent breastfeeding practices by stimulating the release of prolactin, enabling the mother to produce more milk [11]. Yet, only two-thirds of mothers in Nepal are reported to exclusively breastfeed their infants in the past $24 \mathrm{~h}$ (66.1\%) [12]. Concerns exist that, in Nepal, the prevalence of exclusive breastfeeding in early infancy may be in decline, as indicated by a slight reduction from about 70 to $66 \%$ between consecutive Demographic Health Surveys (DHS) from 2011 to 2016 [13].

In Nepal [14], elsewhere in South Asia [15-19] and in other regions [20, 21], colostrum may often be discarded, despite nutritional and immunological benefits it confers to newborns [22], and replaced by prelacteal feeds. Prelacteal feeding not only displaces breastmilk, but also can disrupt the priming of the gastrointestinal tract [23] and may introduce pathogens that increase the risk of illness [24]. Consequent delay in establishing breastfeeding has been shown to predispose infants to a higher risk of mortality in a dose response fashion [3].

In South Asia, including Nepal, despite the increased policy and programmatic investment in behavior change communication to promote optimal feeding practices for infants [25], achieving the targets set by WHO is proving to be challenging [26]. Small area studies have been conducted in Nepal to identify factors related to infant feeding practices, mothers' knowledge on how long a child should be given only breast milk, perceptions about the benefits of breastfeeding, socioeconomic status, and mothers' education [27-29] that may help guide more effective breastfeeding promotion. However, there remains uncertainty about the generalizability of these findings nationally. The present paper presents prevalence estimates for four breastfeeding practices as assessed in a nationally representative sample of infants $(<12$ months of age) in Nepal and examines factors that are associated with these feeding practices at individual, household and community levels.

\section{Methods}

\section{Study design}

Data used for this analysis was collected during a national survey (the PoSHAN Community Study) conducted from May to July 2013. The design of the study is described in detail elsewhere [30]. In brief, systematic random sampling following a random start was carried out to select village development committees (VDCs) from a West to East listing of all contiguous VDCs in each agro-ecological zone. Seven VDCs, each from different districts, across each zone (a total of 21 VDCs in 21 districts) were selected. Wards were listed by population size in each VDC $(n=9)$ from which three were systematically selected following a random start. In total, 63 wards were sampled $(21 \times 3)$, in which all households were visited. The study districts are shown in Fig. 1. The households were eligible for the study if there were children less than 5 years of age or women without children who were married within the past 2 years. Heads of household and mothers were consented and invited to participate in the survey. Information was collected on household characteristics, mothers and children under 5 years of age. However, for the present analysis of breastfeeding practices and risk factors, we include data only from households with infants under 12 months of age at the time of the survey to minimize recall bias with respect to early infant feeding practices that may exist among mothers of older children [31].

\section{Data collection}

In each sampled VDC, 21 field teams, each consisting of three experienced interviewers and one supervisor were hired from a local research firm (New ERA Pvt. Ltd). Field teams were trained and standardized in obtaining informed consent and conducting interviews over a period of a month. Questionnaires were pre-tested across agro-ecological zones and interviews were conducted primarily in Nepali. The final questionnaires were in Nepali and translation was done where required. In certain VDCs, as appropriate, interviews were conducted in Awadhi, Maithilee and Bhojpuri languages. Data collection was monitored in the field by a trained supervisor and quality control team. Household information was obtained by interviewing the head, whereas maternal and child levels of information were obtained by interviewing mothers. Data were collected using paper forms that were checked in the field for legibility and completeness and transmitted to a data entry center in Kathmandu for checking, entry and range and other consistency checks were undertaken.

\section{Outcome variables}

Among infants, field staff inquired about early initiation of breastfeeding (within $1 \mathrm{~h}$ of birth), feeding of colostrum and any prelacteal feeds, and predominant breastfeeding as study outcomes. We used predominant breastfeeding instead of exclusive breastfeeding as data was not collected on intake of water-based fluids by infants in the past 7 days [32]. Use of predominant breastfeeding as an indicator is 


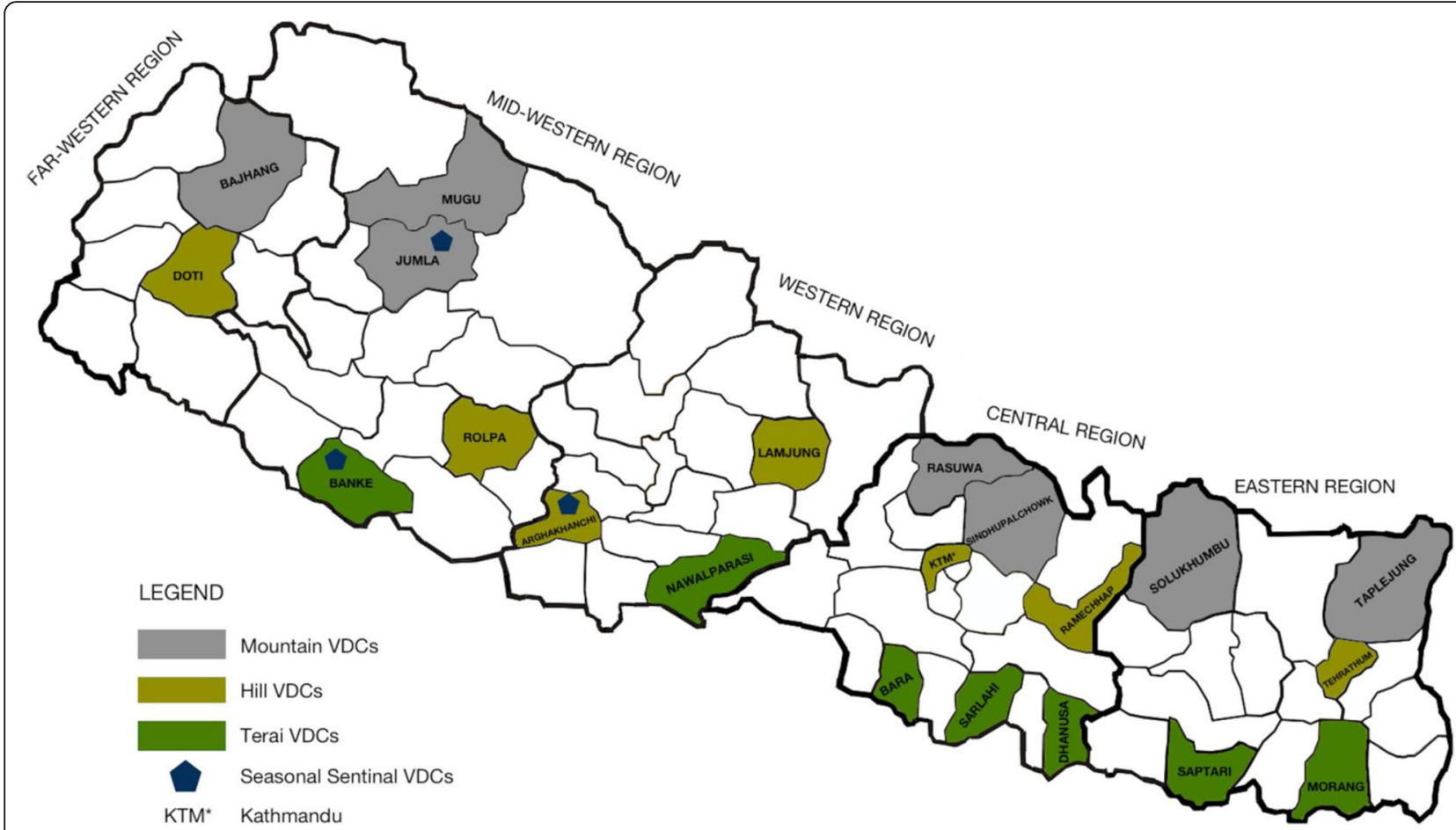

Fig. 1 PoSHAN Community Study districts, Nepal, 2013 (adapted with permission from [30])

helpful to understand breastfeeding practices in the absence of an exclusive breastfeeding indicator. However, the rates from these two exclusive breastfeeding and predominant breastfeeding indicators cannot be directly comparable and one should clearly explain which rate is being reported. The definitions and measurement approaches for the outcome variables were based on the WHO Infant and Young Child Feeding (IYCF) indicator guide, with the exception of feeding colostrum and prelacteal feeds, which we appended to adopted WHO indicator variables [32]. Definitions of these outcomes are provided below:

\section{Breastfeeding within one hour of birth}

Mothers of infants were asked how soon after birth the child was put to the breast and enumerators coded responses into four categories $(<1 \mathrm{~h}, 1 \mathrm{~h}$ to $<24 \mathrm{~h}$, two or more days, never breastfed). We dichotomized these responses using a cutoff of $<1 \mathrm{~h}$, for consistency with the WHO indicator.

\section{Feeding Colostrum}

Colostrum was defined as the first yellowish human breast milk produced after giving birth. Mothers were asked whether the child was fed colostrum.

\section{Prelacteal feeds}

Prelacteal feeds were defined as foods or drinks other than human breast milk given to newborns in the first 3 days of life. Mothers giving any prelacteal feeds in the first 3 days of life were categorized as prelacteal feeders.

\section{Predominant breastfeeding}

WHO defines predominant breastfeeding as a condition where a child $<6$ months of age is mainly breastfed, not fed solid/semi-solid foods, infant formula or non-human milk, and may or may not have received water-based fluids (water, water-based drinks, juice, ORS, ritual fluids, vitamins/minerals/medicines) in the past $24 \mathrm{~h}$. However, in this study the recall period was the past 7 days. Also, as this practice is age-dependent, analysis was stratified by age, and restricted to infants $<6$ months of age. Because we did not capture feeding information for the entire first 6 months of life, there is no overlap with prelacteal feeding.

\section{Covariates}

To explore determinants of the breastfeeding practices, we categorized explanatory variables into child, maternal, household, and community-level factors. The selection of these variables was informed by a review of the literature and factors that have been shown or hypothesized to be associated with breastfeeding practices [33, 34].

Child-level factors included gender, age and birth order (first born child vs second or later born child) which were treated as categorical variables. 
Maternal-level factors included age, educational attainment, occupation, antenatal care (ANC) and postnatal care visit, knowledge about recommended breastfeeding practices and women's empowerment. We developed a women's empowerment variable based on a simple 14 item scale of participation in critical household decisions related to expenditures, production, parenting, and autonomy. This was dichotomized at the upper 25th percentile. Mothers who were in the upper 25 percentile with a score of $>=9$ were considered more empowered. All the variables were treated as categorical.

Household-level factors included presence of father in the household; father's education and occupation, household head's gender, education and occupation; ethnicity/ caste; household cultivable land size and wealth quintiles. Principal component analysis was conducted based on a list of durable asset and land ownership using the method described by Ruestein and colleagues [35]. Quintiles of this scale were then created.

Community-level factors included agro-ecological zone (Mountain, Hills and Terai) and VDC infrastructure. A VDC was considered more developed if it had one of the following: presence of paved roads, $\mathrm{PHC} /$ hospitals, permanent bazar or secondary/higher secondary school. All the variables were treated as categorical.

\section{Statistical analysis}

We used prevalence ratios (PR), a measure that is analogous to the risk ratios of cohort studies, to evaluate the associations between determinants and breastfeeding practices [36, 37]. Prevalence ratios and 95\% confidence intervals (95\% CI) were derived using Poisson regression with robust variance to account for clustering by wards [38, 39]. In all multivariable adjusted models, we included mother's education and visit by female community health volunteers (FCHVs) for antenatal care as we identified these variables a priori as important potential covariates because mother's education is an important predictor of breastfeeding practices [40] and FCHVs are heavily involved in community based infant and young child feeding programs [41]. We then used a two-step-approach to make decisions about the selection of additional variables. Unadjusted relationships were first examined (Model 1), and those variables with a $p$ value $<0.2$ were retained in the first set of multivariable models (Model 2), that were run separately for each grouping of covariates; i.e., by levels of child, maternal, household and agro-ecological zone. The final multivariable model (Model 3) included only those variables that had a $p$ value $<0.2$ in Model 2. The threshold used to determine statistical significance for interpretation of all models was a $p$ - value $<0.05$. Only variables retained in each model are presented in tables or the results.
All statistical analyses were performed using Stata version 13.1 (StataCorp, Texas).

\section{Ethical approval}

Participants were briefed about the purpose and assessment activities of the study. Participation was voluntary and agreement to participate was documented as an oral consent. As some of the respondents were illiterate, we could not use informed written consent. Ethical approval for the study was provided by the Nepal Health Research Council, an autonomous body, under the Ministry of Health, Government of Nepal, and the Institutional Review Board at the Johns Hopkins Bloomberg School of Public Health, Baltimore, MD.

\section{Results \\ General characteristics of the participants and breastfeeding practices}

Mothers of 1015 infants were interviewed. Slightly more than half of the infants were male (53.5\%) and aged 6 to 11.9 months $(54.9 \%)$. Most interviewed mothers were 20 to 29 years of age $(69.0 \%)$, while $14.7 \%$ were adolescents (15 to 19 years of age). Nearly half of the mothers (47.4\%) had never attended school, $13.3 \%$ had some primary education and $39.8 \%$ had at least secondary education. Three quarters of mothers (76.4\%) were not formally employed and $13.5 \%$ were employed in agriculture. More than half of the infants resided in the Terai (58.8\%), and a quarter in Hills $(25.4 \%)$ with the remainder $(15.8 \%)$ in the Mountains (Table 1$)$.

Breast milk was introduced within $1 \mathrm{~h}$ of birth in $41.8 \%$ of infants. One-third of infants (32.7\%) were reported to have received prelacteal feeds as their first food, $83.5 \%$ were fed colostrum, and predominant breastfeeding (PBF) was practiced by $57.2 \%$ of interviewed mothers infants less than 6 months of age (Table 1).

\section{Determinants of breastfeeding within one hour of birth (Table 2)}

In multivariable adjusted models exploring predictors of breastfeeding within $1 \mathrm{~h}$ of birth, associations with maternal age were apparent: compared with infants of younger mothers $(<20 \mathrm{y})$ those born to mothers $20-29$ and $\geq 30$ years of age were $19 \%$ (adjusted prevalence ratio [APR] 0.81; 95\% CI 0.68, 0.95) and 39\% (APR 0.61; 95\% CI $0.43,0.87$ ) less likely to have reported breastfeeding within an hour after birth. Mothers who had agriculture as an occupation were also $28 \%$ more likely to have breastfed their children within one hour of birth compared to mothers who were unemployed (APR 1.28; 95\% CI 1.02, 1.60). 
Table 1 General characteristics of study population and explanatory factors

\begin{tabular}{ll}
$n(\%)$ \\
\hline
\end{tabular}

\begin{tabular}{|c|c|}
\hline \multicolumn{2}{|l|}{ Child level factors $(n=1015)$} \\
\hline \multicolumn{2}{|l|}{ Child's gender } \\
\hline Male & $543(53.5)$ \\
\hline Female & $472(46.5)$ \\
\hline Child's age (in months), mean (SD) & $5.9(3.4)$ \\
\hline \multicolumn{2}{|l|}{ Child's age (in months) } \\
\hline $0-5.9$ & $458(45.1)$ \\
\hline $6-11.9$ & $557(54.9)$ \\
\hline \multicolumn{2}{|l|}{ Birth order } \\
\hline First born child & $540(53.2)$ \\
\hline Second or later born child & $475(46.8)$ \\
\hline \multicolumn{2}{|l|}{ Maternal level factors $(n=1015)$} \\
\hline Mother's age (in years), mean (SD) & $24.4(5.4)$ \\
\hline \multicolumn{2}{|l|}{ Mother's age (in years) } \\
\hline $15-19.9$ & $149(14.7)$ \\
\hline $20-29.9$ & $700(69.0)$ \\
\hline$>=30$ & $166(16.4)$ \\
\hline \multicolumn{2}{|l|}{ Mother's education } \\
\hline None & $481(47.4)$ \\
\hline Some primary & $135(13.3)$ \\
\hline Secondary and above & $398(39.3)$ \\
\hline \multicolumn{2}{|l|}{ Mother's occupation } \\
\hline Unemployed & $775(76.4)$ \\
\hline Agriculture & $137(13.5)$ \\
\hline Other & $103(10.2)$ \\
\hline
\end{tabular}

Visit by FCHV for ANC

$\begin{array}{ll}\text { No } & 913(90.0) \\ \text { Yes } & 102(10.1)\end{array}$

Visit by more highly trained healthcare providers ${ }^{a}$ for ANC

$\begin{array}{ll}\text { No } & 976(96.2) \\ \text { Yes } & 39(3.8)\end{array}$

Visit to health facilities for ANC

No

$390(38.4)$

Yes

625 (61.6)

Visit by FCHV for postnatal care

$$
\begin{array}{ll}
\text { No } & 907(89.4) \\
\text { Yes } & 108(10.6)
\end{array}
$$

Visit by more highly trained healthcare providers ${ }^{\text {a }}$ for PNC

$$
\text { No }
$$

$963(94.9)$

Yes

$52(5.1)$

Visit to health facilities for postnatal care

$$
\text { No }
$$

Yes
Table 1 General characteristics of study population and explanatory factors (Continued)

\begin{tabular}{ll}
\hline & $n(\%)$ \\
\hline Maternal knowledge present on: & \\
Exclusive breastfeeding for infants up to 6 months of age \\
$\quad$ No & $368(36.3)$ \\
Yes & $647(63.7)$ \\
Breastfeeding for children during diarrhea & \\
No & $809(79.7)$ \\
Yes & $206(20.3)$ \\
Mothers' empowerment (mean, SD) & $5.5(3.1)$ \\
Mothers' empowerment (scale: 0-14, Median =5) & \\
$<=8$ (less empowered) & $830(81.8)$ \\
$>=9$ (more empowered) & $185(18.2)$
\end{tabular}

Household level factors $(n=1015)$

Presence of father at home

No

$325(32.0)$

Yes

$690(68.0)$

Father's education (among those present at home)

None

$154(22.3)$

Some primary

$149(21.6)$

Secondary and above

$387(56.1)$

Household head's gender

Male

808 (79.6)

Female

$207(20.4)$

Household head's education

None

$485(47.8)$

Some primary

$191(18.8)$

Secondary and above

$339(33.4)$

Household head's occupation

Unemployed ${ }^{\mathrm{a}}$

$122(12.0)$

Wage employment

$190(18.7)$

Business/self-employment

$212(20.9)$

Salaried worker

$101(10.0)$

Agriculture

389 (38.4)

Ethnicity/Caste

Upper caste (Brahmins, chhetris) 218 (21.5)

Disadvantaged non-dalit Terai caste 341 (33.6)

Janajatis 228 (22.5)

Lower caste (Dalits, religious minorities) 228 (22.5)

Household wealth quintile

$\begin{array}{ll}1 \text { (Poorest) } & 203(20.0) \\ 2 & 199(19.6) \\ 3 & 204(20.1) \\ 4 & 206(20.3) \\ 5 \text { (Richest) } & 203(20.0)\end{array}$


Table 1 General characteristics of study population and explanatory factors (Continued)

\begin{tabular}{|c|c|}
\hline & $n(\%)$ \\
\hline \multicolumn{2}{|c|}{ Father's occupation (among those present at home) } \\
\hline Unemployed ${ }^{\text {a }}$ & $19(2.8)$ \\
\hline Wage employment & $195(28.3)$ \\
\hline Business/self-employment & $178(25.8)$ \\
\hline Salaried worker & $124(18.0)$ \\
\hline Agriculture & $174(25.2)$ \\
\hline \multicolumn{2}{|l|}{ Cultivable land size (in $\mathrm{Ha}$ ) } \\
\hline Landless $(<0.1)$ & $424(41.8)$ \\
\hline Small size $(>=0.1 \&<0.5)$ & $261(25.7)$ \\
\hline Large size $(>=0.5)$ & $330(32.5)$ \\
\hline \multicolumn{2}{|l|}{ Contextual factors $(n=1015)$} \\
\hline \multicolumn{2}{|l|}{ Agro-ecological zones } \\
\hline Mountain & $160(15.8)$ \\
\hline Hill & $258(25.4)$ \\
\hline Terai & $597(58.8)$ \\
\hline \multicolumn{2}{|c|}{ Ward infrastructure is more developed } \\
\hline No & $502(49.5)$ \\
\hline Yes & $513(50.5)$ \\
\hline \multicolumn{2}{|l|}{ Breastfeeding practices } \\
\hline \multicolumn{2}{|l|}{ Prelacteal feeds given } \\
\hline Not fed & $677(67.3)$ \\
\hline Fed & $329(32.7)$ \\
\hline \multicolumn{2}{|c|}{ Breastfed within one hour after birth } \\
\hline No & $588(58.2)$ \\
\hline Yes & $423(41.8)$ \\
\hline \multicolumn{2}{|l|}{ Colostrum fed } \\
\hline No & $167(16.5)$ \\
\hline Yes & $844(83.5)$ \\
\hline \multicolumn{2}{|c|}{ Predominant breastfeeding (children $<6$ months) $(n=458)$} \\
\hline No & $196(42.8)$ \\
\hline Yes & $262(57.2)$ \\
\hline
\end{tabular}

a"More highly trained healthcare providers" includes other govt health workers (MCHW/VHW, HA/AHW, Nurse/Midwife), doctors/pharmacists and NGO health workers; "Unemployed" includes student, non-earning occupation as well as non-working

\section{Determinants of colostrum feeding (Table 3)}

Several factors were associated with a slight but significant increased likelihood of feeding the newborn infant colostrum in multivariable adjusted models, including maternal age 20-29 y (vs. age $<20$ y), greater women's empowerment, a reproductive history that included an abortion in their lifetime, a large land holding and household wealth classified to be in the upper 40th percent of the nationally compiled index (vs. in the lowest fifth). Infants born to mothers in households where the heads were salaried workers or involved in agriculture were 9\% less likely to be given colostrum (APR 0.91; 95\% CI 0.84, 0.98) compared to the newborns into households whose head was unemployed. Newborns in the Terai were $10 \%$ less likely to receive colostrum than those born in the mountains (APR 0.90; 95\% CI 0.83, 0.97).

\section{Determinants of prelacteal feeding (Table 4)}

Second or later born infants were $31 \%$ less likely than first born infants to have received prelacteal feeds (APR 0.69; 95\% CI 0.55, 0.87). Infants born in the Terai were 2.7 times more likely to have been fed prelacteal feeds than those in the mountains (APR 2.72; 95\% CI 1.67, $4.45)$. A history of any antenatal care visit was associated with a greater chance of a mother providing prelacteal feeds, especially visits by healthcare workers other than the local FCHV compared to the mothers who did not go for antenatal care visit (APR 1.43; 95\% CI 1.11, 1.84).

\section{Determinants of predominant breastfeeding under six months (Table 5)}

Compared to infants $<2$ months of age, infants of age 2 to 3.9 months and 4 to 5.9 months were $24 \%$ (APR 0.86; 95\% CI $0.75,0.98$ ) and 43\% (APR 0.57; 95\% CI 0.42, 0.77 ) less likely to be predominantly breastfed, respectively. Children of mothers who visited health facilities for antenatal care visits were 19\% (APR 1.19; 95\% CI $1.02,1.38)$ more likely to predominantly breastfeed than those who did not visit health facilities for antenatal care visit. Compared to women without knowledge, those women who had knowledge of exclusive breastfeeding for infants up to 6 months of age were 19\% more likely to report predominantly breastfeeding their infants (APR 1.19; 95\% CI 1.01, 1.39). However, paradoxically, those with knowledge of the need to breastfeed through diarrheal episodes were $20 \%$ less likely to predominantly breastfeed than those without the knowledge (APR 0.80; 95\% CI 0.66, 0.97). Children from lower caste families were $47 \%$ more likely to predominantly breastfeed compared to the upper caste families (APR 1.47; 95\% CI $1.02,2.12)$. Those infants in the second lowest fifth of the constructed wealth index had a $32 \%$ lower chance of predominant breastfeeding compared with infants born into the poorest $20 \%$ of households (APR 0.68; $95 \% \mathrm{CI}$ $0.51,0.91)$. Compared to the children living in the mountains, infants born in households in the Hills were $33 \%$ less likely to be predominantly breastfed (APR 0.67; 95\% CI 0.49, 0.93) (Table 5).

\section{Coexistence of breastfeeding practice indicators}

This study also assessed inter-relationships between breastfeeding practices. Compared to infants not fed prelacteal feeds, infants given prelacteal feeds were $51 \%$ less likely to be breastfed within the first hour of birth (APR 0.49; 95\% CI 0.36, 0.66) and 55\% less likely to be 
Table 2 Determinants of breastfeeding within one hour of birth among infants in Nepal, 2013 a,b,c

\begin{tabular}{|c|c|c|c|c|}
\hline Determinants & $n(\%)$ & $\begin{array}{l}\text { Breastfed within an hour } \\
n(\%)\end{array}$ & $\begin{array}{l}\text { Model } 1 \\
\text { (Unadjusted PR) } \\
\text { PR }(95 \% \mathrm{Cl})\end{array}$ & $\begin{array}{l}\text { Model } 3^{\text {d }} \\
\text { (Adjusted PR) } \\
\text { APR (95\% Cl) }\end{array}$ \\
\hline Overall & $1011(100)$ & $423(41.8)$ & & \\
\hline \multicolumn{5}{|l|}{ Child factors } \\
\hline \multicolumn{5}{|l|}{ Child's gender } \\
\hline Male & $541(53.5)$ & $222(41.0)$ & 1.00 & - \\
\hline Female & $470(46.5)$ & 201(42.8) & $1.03(0.91-1.18)$ & \\
\hline \multicolumn{5}{|l|}{ Child's birth order } \\
\hline First born & $538(53.2)$ & $210(39.0)$ & 1.00 & - \\
\hline Second or later born & $473(46.8)$ & $213(45.0)$ & $1.18(1.00,1.38)^{*}$ & \\
\hline \multicolumn{5}{|l|}{ Child fed colostrum } \\
\hline No & $167(16.5)$ & $54(32.3)$ & 1.00 & 1.00 \\
\hline Yes & $844(83.5)$ & $369(43.7)$ & $1.32(1.07,1.64)^{*}$ & $1.26(1.04,1.54)^{*}$ \\
\hline \multicolumn{5}{|l|}{ Child fed prelacteal feeds } \\
\hline No & $677(67.3)$ & $343(50.9)$ & 1.00 & 1.00 \\
\hline Yes & $329(32.7)$ & $78(23.7)$ & $0.47(0.35,0.63)^{* *}$ & $0.49(0.36,0.66)^{* *}$ \\
\hline \multicolumn{5}{|c|}{ Predominant breastfeeding (Infant $<6 \mathrm{mo}$ ) } \\
\hline No & $196(42.8)$ & $75(38.3)$ & 1.00 & - \\
\hline Yes & $262(57.2)$ & $130(50.0)$ & $1.33(1.01,1.74)^{*}$ & \\
\hline \multicolumn{5}{|l|}{ Maternal factors } \\
\hline \multicolumn{5}{|l|}{ Mother's education } \\
\hline None & $479(47.4)$ & $200(41.8)$ & 1.00 & 1.00 \\
\hline Some primary & $135(13.4)$ & $53(39.3)$ & $0.93(0.72,1.2)$ & $0.91(0.70-1.19)$ \\
\hline Secondary and above & $396(39.2)$ & $170(42.9)$ & $1.00(0.87,1.16)$ & $1.01(0.84-1.21)$ \\
\hline \multicolumn{5}{|l|}{ Mother's age (in years) } \\
\hline $15-19.9$ & $149(14.7)$ & $68(45.6)$ & 1.00 & 1.00 \\
\hline $20-29.9$ & $697(68.9)$ & $299(42.9)$ & $0.93(0.79,1.09)$ & $0.81(0.68-0.95)^{*}$ \\
\hline$\geq 30$ & $165(16.3)$ & $56(33.9)$ & $0.72(0.53,0.97)^{*}$ & $0.61(0.43-0.87)^{*}$ \\
\hline \multicolumn{5}{|l|}{ Mother's occupation } \\
\hline Unemployed & $773(76.5)$ & $305(39.5)$ & 1.00 & 1.00 \\
\hline Agriculture & $136(13.5)$ & $72(52.9)$ & $1.31(1.04,1.64)^{*}$ & $1.28(1.02-1.60)^{*}$ \\
\hline Other employment ${ }^{\mathrm{e}}$ & $102(10.1)$ & $46(45.1)$ & $1.10(0.85,1.43)$ & $1.09(0.83-1.42)$ \\
\hline \multicolumn{5}{|l|}{ Visit by FCHVs for ANC } \\
\hline No & $909(89.9)$ & $382(42)$ & 1.00 & \\
\hline Yes & $102(10.1)$ & $41(40.2)$ & $0.98(0.78,1.24)$ & $0.99(0.77-1.27)$ \\
\hline \multicolumn{5}{|c|}{ Visit by FCHVs for postnatal care } \\
\hline No & $903(89.3)$ & $371(41.1)$ & 1.00 & 1.00 \\
\hline Yes & $108(10.7)$ & $52(48.2)$ & $1.17(0.94,1.45)$ & $1.12(0.91-1.37)$ \\
\hline \multicolumn{5}{|c|}{ Visit by more highly trained healthcare providers ${ }^{\mathrm{e}}$ for postnatal care } \\
\hline No & $959(94.9)$ & $408(42.5)$ & 1.00 & 1.00 \\
\hline Yes & $52(5.1)$ & $15(28.9)$ & $0.69(0.49,0.99)^{*}$ & $0.72(0.49-1.05)$ \\
\hline \multicolumn{5}{|c|}{ Maternal knowledge on exclusive breastfeeding for infants up to 6 months of age } \\
\hline No & $368(36.4)$ & $136(37)$ & 1.00 & 1.00 \\
\hline Yes & $643(63.6)$ & $287(44.6)$ & $1.17(0.97,1.41)$ & $1.19(0.99-1.44)$ \\
\hline
\end{tabular}

Number of live births given 
Table 2 Determinants of breastfeeding within one hour of birth among infants in Nepal, $2013^{a, b, c}$ (Continued)

\begin{tabular}{|c|c|c|c|c|}
\hline Determinants & $n(\%)$ & $\begin{array}{l}\text { Breastfed within an hour } \\
n(\%)\end{array}$ & $\begin{array}{l}\text { Model } 1 \\
\text { (Unadjusted PR) } \\
\text { PR (95\% Cl) }\end{array}$ & $\begin{array}{l}\text { Model } 3^{\mathrm{d}} \\
\text { (Adjusted PR) } \\
\text { APR }(95 \% \mathrm{Cl})\end{array}$ \\
\hline 1 & $380(37.7)$ & $149(39.2)$ & 1.00 & 1.00 \\
\hline$>=2$ & $629(62.3)$ & $274(43.6)$ & $1.12(0.96,1.30)$ & $1.11(0.85-1.43)$ \\
\hline \multicolumn{5}{|l|}{ Household factors } \\
\hline \multicolumn{5}{|l|}{ Ethnicity/Caste } \\
\hline Upper caste & $216(21.4)$ & $104(48.2)$ & 1.00 & 1.00 \\
\hline Disadvantaged non-dalit Terai caste & $341(33.7)$ & $137(40.2)$ & $0.95(0.76,1.20)$ & $1.02(0.81-1.27)$ \\
\hline Janajatis & $227(22.5)$ & $87(38.3)$ & $0.83(0.67,1.04)$ & $0.91(0.73-1.14)$ \\
\hline Lower caste $e^{e}$ & $227(22.5)$ & 95 (41.9) & $0.93(0.74,1.17)$ & $0.95(0.76-1.19)$ \\
\hline \multicolumn{5}{|l|}{ Household wealth quintile } \\
\hline 1 (Poorest) & $202(20)$ & $89(44.1)$ & 1.00 & 1.00 \\
\hline 2 & $198(19.6)$ & $79(39.9)$ & $0.92(0.73,1.14)$ & $0.94(0.75-1.18)$ \\
\hline 3 & $204(20.2)$ & $93(45.6)$ & $1.03(0.8,1.33)$ & $1.07(0.82-1.38)$ \\
\hline 4 & $204(20.2)$ & $93(45.6)$ & $1.05(0.82,1.34)$ & $1.07(0.79-1.45)$ \\
\hline 5 (Richest) & $203(20.1)$ & $69(34.0)$ & $0.77(0.57,1.06)$ & $0.79(0.55-1.12)$ \\
\hline \multicolumn{5}{|l|}{ Occupation of household head } \\
\hline Unemployed $^{e}$ & $122(12.1)$ & $51(41.8)$ & 1.00 & 1.00 \\
\hline Wage employment & $190(18.8)$ & $83(43.7)$ & $1.07(0.83,1.39)$ & $1.03(0.81-1.33)$ \\
\hline Business/self-employment & $210(20.8)$ & $72(34.3)$ & $0.82(0.58,1.17)$ & $0.80(0.57-1.13)$ \\
\hline Salaried worker & $101(10)$ & $57(56.4)$ & $1.32(1.01,1.73)^{*}$ & $1.27(0.94-1.71)$ \\
\hline Agriculture & $387(38.3)$ & $160(41.3)$ & $0.99(0.73,1.34)$ & $0.88(0.65-1.19)$ \\
\hline \multicolumn{5}{|l|}{ Contextual factors } \\
\hline \multicolumn{5}{|l|}{ Agro-ecological zones } \\
\hline Mountain & $158(15.6)$ & $77(48.7)$ & - & 1.00 \\
\hline Hill & $256(25.3)$ & $111(43.4)$ & & $1.00(0.79-1.26)$ \\
\hline Terai & $597(59.1)$ & 235(39.4) & & $1.03(0.78-1.36)$ \\
\hline
\end{tabular}

${ }^{\mathrm{a}}$ For interpretation purposes, a PR $>1$ indicates children are more likely to be breastfed within an hour of birth and PR $<1$ indicates children are less likely $b_{*} P$-value $<0.05,{ }^{* *} P$-value $<0.001$

'(Model 2 shown in Additional file 1)

${ }^{\mathrm{d}}$ Model 3 included mother's education and visit by FCHVs for ANC as a priori covariates plus all variables that were significant $(p<0.2)$ in the first set of multivariable models

e"Other employment" included wage employment, salaried worker and Business/self-employment. "More highly trained healthcare providers" includes government health workers (MCHW/VHW, HA/AHW, Nurse/Midwife), doctors/pharmacists and NGO health workers. "Lower caste" includes Dalits and religious minorities. "Unemployed" includes student, non-earning occupation as well as non-working

predominantly breastfed through 6 months of age (APR 0.45 ; $95 \%$ CI $0.32,0.62$ ). Infants reported to have received colostrum were $26 \%$ more likely to have started breastfeeding within an hour of birth (APR 1.26; 95\% CI $1.04,1.54$ ) compared to those who did not receive colostrum. Compared to infants who were not breastfed within an hour of birth, infants breastfed within $1 \mathrm{~h}$ of birth have $50 \%$ less chance of being fed prelacteal feeds (APR 0.50; 95\% CI 0.37, 0.67).

\section{Discussion}

This study profiles the prevalence, quality and determinants of breastfeeding practices in a national sample of infants in Nepal. While breastfeeding is nearly universal, most mothers delay introduction of breastmilk by an hour or more after delivery. Our estimates of the percentage of mothers introducing breastmilk within $1 \mathrm{~h}$ of birth was lower ( $41.8 \%$ vs. $54.9 \%)$, and prelacteal feeding slightly higher (32.7 vs. 28.6\%) than reported in the 2016 Demographic and Health Survey (DHS) . This difference might be due, in part, to different recall periods, with the DHS including children born in the 2 years preceding the survey without regard to vital status at the time of interview. Local area sampling variability and variation in the way questions were coded may also lead to differences in estimates. As the DHS does not report colostrum feeding, our estimate that $83.5 \%$ of mothers fed colostrum at some time in the early breastfeeding 
Table 3 Determinants of feeding colostrum among infants in Nepal, 2013 a,b,c

\begin{tabular}{|c|c|c|c|c|}
\hline Determinants & $n$ & $\begin{array}{l}\text { Fed colostrum, } \\
n(\%)\end{array}$ & $\begin{array}{l}\text { Model } 1 \\
\text { (Unadjusted PR) } \\
\text { PR (95\% Cl) }\end{array}$ & $\begin{array}{l}\text { Model } 3^{d} \\
\text { (Adjusted PR) } \\
\text { APR }(95 \% \text { Cl) }\end{array}$ \\
\hline Overall & 1011 & $844(83.5)$ & & \\
\hline \multicolumn{5}{|l|}{ Child factors } \\
\hline \multicolumn{5}{|l|}{ Child's gender } \\
\hline Male & 541 & $448(82.8)$ & 1.00 & \multirow[t]{2}{*}{-} \\
\hline Female & 470 & 396(84.3) & $1.01(0.96-1.06)$ & \\
\hline \multicolumn{5}{|l|}{ Child's birth order } \\
\hline First born & 538 & $455(84.6)$ & 1.00 & \multirow[t]{2}{*}{-} \\
\hline Second or later born & 473 & $389(82.2)$ & $0.99(0.93-1.06)$ & \\
\hline \multicolumn{5}{|c|}{ Breastfed within one hour of birth } \\
\hline No & 588 & $475(80.8)$ & 1.00 & 1.00 \\
\hline Yes & 423 & $369(87.2)$ & $1.07(1.01,1.13)^{*}$ & $1.06(1.01,1.11)^{*}$ \\
\hline \multicolumn{5}{|l|}{ Child fed prelacteal feeds } \\
\hline No & 674 & $584(86.7)$ & 1.00 & 1.00 \\
\hline Yes & 329 & $255(77.5)$ & $0.92(0.84,1.00)^{*}$ & $0.92(0.86,0.99)^{*}$ \\
\hline \multicolumn{5}{|c|}{ Predominant breastfeeding (Infant $<6 \mathrm{mo}$ ) } \\
\hline No & 196 & $166(84.7)$ & 1.00 & \multirow[t]{2}{*}{-} \\
\hline Yes & 260 & 217 (83.5) & $1.00(0.94,1.05)$ & \\
\hline \multicolumn{5}{|l|}{ Maternal factors } \\
\hline \multicolumn{5}{|l|}{ Mother's education } \\
\hline None & 479 & $373(77.9)$ & 1.00 & 1.00 \\
\hline Some primary & 135 & $115(85.2)$ & $1.08(1-1.17)$ & $1.05(0.96-1.14)$ \\
\hline Secondary and above & 396 & $355(89.7)$ & $1.12(1.06-1.19)^{* *}$ & $1.04(0.97-1.12)$ \\
\hline \multicolumn{5}{|l|}{ Mother's age (in years) } \\
\hline 15-19.9 & 149 & $113(75.8)$ & 1.00 & 1.00 \\
\hline $20-29.9$ & 697 & $595(85.4)$ & $1.12(1.02-1.23)^{*}$ & $1.09(1.00-1.19)^{*}$ \\
\hline$\geq 30$ & 165 & $136(82.4)$ & $1.05(0.93-1.19)$ & $1.07(0.94-1.22)$ \\
\hline \multicolumn{5}{|l|}{ Visit by FCHVs for ANC } \\
\hline No & 909 & 760 (83.6) & 1.00 & 1.00 \\
\hline Yes & 102 & $84(82.4)$ & $1.01(0.91-1.13)$ & $1(0.91-1.11)$ \\
\hline \multicolumn{5}{|c|}{ Visit by more highly trained healthcare providers ${ }^{\mathrm{e}}$ for ANC } \\
\hline No & 972 & $816(84.0)$ & 1.00 & 1.00 \\
\hline Yes & 39 & $28(71.8)$ & $0.91(0.8-1.02)$ & $0.94(0.86-1.03)$ \\
\hline \multicolumn{5}{|c|}{ Visit to health facilities for ANC } \\
\hline No & 388 & $308(79.4)$ & 1.00 & 1.00 \\
\hline Yes & 623 & $536(86.0)$ & $1.08(1.00-1.17)$ & $1.07(0.98-1.16)$ \\
\hline \multicolumn{5}{|c|}{ Maternal knowledge on exclusive breastfeeding for infants up to 6 months of age } \\
\hline No & 368 & $285(77.5)$ & 1.00 & 1.00 \\
\hline Yes & 643 & $559(86.9)$ & $1.08(1.02-1.15)^{*}$ & $1.04(0.98-1.09)$ \\
\hline \multicolumn{5}{|c|}{ Women's empowerment (scale: $0-14, \mathrm{Md}=5$ ) } \\
\hline$\leq 8$ (less empowered) & 826 & $677(82.0)$ & 1.00 & 1.00 \\
\hline$\geq 9$ (more empowered) & 185 & $167(90.3)$ & $1.07(1.00-1.14)^{*}$ & $1.08(1.01-1.15)^{*}$ \\
\hline \multicolumn{5}{|l|}{ Had abortions in lifetime } \\
\hline No & 972 & 806 (82.9) & 1.00 & 1.00 \\
\hline
\end{tabular}


Table 3 Determinants of feeding colostrum among infants in Nepal, 2013 $3^{\mathrm{a}, \mathrm{b}, \mathrm{c}}$ (Continued)

\begin{tabular}{|c|c|c|c|c|}
\hline Determinants & $n$ & $\begin{array}{l}\text { Fed colostrum, } \\
n(\%)\end{array}$ & $\begin{array}{l}\text { Model } 1 \\
\text { (Unadjusted PR) } \\
\text { PR }(95 \% \mathrm{Cl})\end{array}$ & $\begin{array}{l}\text { Model } 3^{\text {d }} \\
\text { (Adjusted PR) } \\
\text { APR ( } 95 \% \text { Cl) }\end{array}$ \\
\hline Yes & 39 & $38(97.4)$ & $1.12(1.06-1.19)^{* *}$ & $1.10(1.02-1.17)^{*}$ \\
\hline \multicolumn{5}{|c|}{ Had miscarriage/stillbirths in lifetime } \\
\hline No & 847 & $713(84.2)$ & 1.00 & 1.00 \\
\hline Yes & 164 & $131(79.9)$ & $0.94(0.88-1.00)$ & $0.93(0.87-1.00)$ \\
\hline \multicolumn{5}{|l|}{ Household factors } \\
\hline \multicolumn{5}{|l|}{ Household head's education } \\
\hline None & 484 & $381(78.7)$ & 1.00 & 1.00 \\
\hline Some primary & 189 & $159(84.1)$ & $1.06(0.98-1.14)$ & $1.01(0.94-1.09)$ \\
\hline Secondary and above & 338 & $304(89.9)$ & $1.12(1.07-1.17)^{* *}$ & $1.05(1.00-1.10)$ \\
\hline \multicolumn{5}{|l|}{ Household wealth quintile } \\
\hline 1 (Poorest) & 202 & $158(78.2)$ & 1.00 & 1.00 \\
\hline 2 & 198 & $156(78.8)$ & $1.02(0.92-1.13)$ & $1(0.90-1.1)$ \\
\hline 3 & 204 & $162(79.4)$ & $1.02(0.94-1.10)$ & $0.97(0.90-1.06)$ \\
\hline 4 & 204 & $183(89.7)$ & $1.17(1.08-1.26)^{* *}$ & $1.09(1.01-1.19)^{*}$ \\
\hline 5 (Richest) & 203 & $185(91.1)$ & $1.17(1.10-1.25)^{* *}$ & $1.08(1.00-1.18)$ \\
\hline \multicolumn{5}{|l|}{ Occupation of household head } \\
\hline Unemployed $^{e}$ & 122 & $107(87.7)$ & 1.00 & 1.00 \\
\hline Wage employment & 190 & $152(80)$ & $0.94(0.86-1.03)$ & $0.96(0.89-1.05)$ \\
\hline Business/self-employment & 210 & $176(83.8)$ & $0.97(0.90-1.05)$ & $0.94(0.88-1.00)$ \\
\hline Salaried worker & 101 & $88(87.1)$ & $0.98(0.91-1.06)$ & $0.91(0.84-0.98)^{*}$ \\
\hline Agriculture & 387 & $320(82.7)$ & $0.94(0.88-1.02)$ & $0.91(0.84-0.98)^{*}$ \\
\hline \multicolumn{5}{|l|}{ Cultivable land size (in $\mathrm{Ha}$ ) } \\
\hline Landless $(<0.1)$ & 421 & $343(81.5)$ & 1.00 & 1.00 \\
\hline Small size $(\geq 0.1 \&<0.5)$ & 260 & $216(83.1)$ & $1(0.93-1.08)$ & $1.06(0.99-1.15)$ \\
\hline Large size $(\geq 0.5)$ & 330 & $285(86.4)$ & $1.07(1.01-1.14)^{*}$ & $1.12(1.05-1.19)^{* *}$ \\
\hline \multicolumn{5}{|l|}{ Contextual factors } \\
\hline \multicolumn{5}{|l|}{ Agro-ecological zones } \\
\hline Mountain & 158 & $144(91.1)$ & - & 1.00 \\
\hline Hill & 256 & $231(90.2)$ & & $0.99(0.93-1.05)$ \\
\hline Terai & 597 & 469 (78.6) & & $0.9(0.83-0.97)^{*}$ \\
\hline \multicolumn{5}{|c|}{ Ward infrastructure is more developed } \\
\hline No & 502 & $394(78.5)$ & 1.00 & 1.00 \\
\hline Yes & 509 & 450 (88.4) & $1.09(1.00-1.18)^{*}$ & $1.04(0.96-1.13)$ \\
\hline
\end{tabular}

aprevalence ratio: a PR $>1$ indicates feeding of colostrum is more likely and PR $<1$ indicates that feeding of colostrum is less likely

$b_{*}^{*} P$-value $<0.05,{ }^{* *} P$-value $<0.001$

${ }^{c}$ (Model 2 shown in Additional file 2)

${ }^{\mathrm{d}}$ Model 3 included mother's education and visit by FCHVs for ANC as a priori covariates plus all variables that were significant $(p<0.2)$ in the first set of multivariable models

e“more highly trained healthcare providers" includes government health workers (MCHW/VHW, HA/AHW, Nurse/Midwife), doctors/pharmacists and NGO health workers. "Unemployed" includes student, non-earning occupation as well as non-working

experience provides a first national estimate of this practice. Due to differences in definitions, our estimates of predominant breastfeeding are not directly comparable to DHS estimates of exclusive breastfeeding, as we did not collect information on intake of fluids and, under an assumption that the transition from exclusive breastfeeding to inclusion of other food items may initially be sporadic and a single $24 \mathrm{~h}$ recall period overestimates the prevalence [42], we used a recall period of 7 days while DHS used a $24 \mathrm{~h}$ recall period. While the lack of information on exclusive breastfeeding prevalence in our population is a study limitation, predominant breastfeeding 
Table 4 Determinants of pre-lacteal feeding among infants in Nepal, 2013 a,b,c

\begin{tabular}{|c|c|c|c|c|}
\hline Determinants & $n$ & Fed prelacteal feeds (\%) & $\begin{array}{l}\text { Model } 1 \text { (Unadjusted PR) } \\
\text { PR ( } 95 \% \text { Cl) }\end{array}$ & $\begin{array}{l}\text { Model } 3^{\text {d }} \text { (Adjusted PR) } \\
\text { APR }(95 \% \text { Cl) }\end{array}$ \\
\hline Overall & 1006 & $329(32.7)$ & & \\
\hline \multicolumn{5}{|l|}{ Child factors } \\
\hline \multicolumn{5}{|l|}{ Child's gender } \\
\hline Male & 537 & 177(33.0) & 1.00 & - \\
\hline Female & 469 & 152(32.4) & $1.03(0.89-1.19)$ & \\
\hline \multicolumn{5}{|l|}{ Child's birth order } \\
\hline First born & 534 & $205(38.4)$ & 1.00 & 1.00 \\
\hline Second or later born & 472 & $124(26.3)$ & $0.65(0.53-0.81)^{* *}$ & $0.72(0.60,0.86)^{* *}$ \\
\hline \multicolumn{5}{|c|}{ Breastfed within one hour of birth } \\
\hline No & 582 & $251(43.1)$ & 1.00 & 1.00 \\
\hline Yes & 421 & $78(18.5)$ & $0.46(0.34,0.62)^{* *}$ & $0.5(0.37,0.67)^{* *}$ \\
\hline \multicolumn{5}{|l|}{ Child fed colostrum } \\
\hline No & 164 & $74(45.1)$ & 1.00 & 1.00 \\
\hline Yes & 839 & $255(30.4)$ & $0.78(0.63,0.96)^{*}$ & $0.78(0.65,0.93)^{*}$ \\
\hline \multicolumn{5}{|c|}{ Predominant breastfeeding (Infant < 6 mo) } \\
\hline No & 195 & $104(53.3)$ & 1.00 & 1.00 \\
\hline Yes & 257 & $43(16.7)$ & $0.49(0.34,0.71)^{* *}$ & $0.51(0.36,0.72)^{* *}$ \\
\hline \multicolumn{5}{|l|}{ Maternal factors } \\
\hline \multicolumn{5}{|l|}{ Mother's education } \\
\hline None & 477 & $156(32.7)$ & 1.00 & 1.00 \\
\hline Some primary & 135 & $46(34.1)$ & $1.06(0.84-1.33)$ & $0.92(0.72-1.17)$ \\
\hline Secondary and above & 393 & $126(32.1)$ & $1.15(0.91-1.46)$ & $0.90(0.70-1.15)$ \\
\hline \multicolumn{5}{|l|}{ Visit by FCHVs for ANC } \\
\hline No & 906 & $285(31.5)$ & 1.00 & 1.00 \\
\hline Yes & 100 & $44(44.0)$ & $1.17(0.92-1.50)$ & $1.14(0.87-1.50)$ \\
\hline \multicolumn{5}{|c|}{ Visit by more highly trained healthcare providers ${ }^{e}$ for ANC } \\
\hline No & 967 & $308(31.9)$ & 1.00 & 1.00 \\
\hline Yes & 39 & $21(53.9)$ & $1.28(0.90-1.81)$ & $1.43(1.11-1.84)^{*}$ \\
\hline \multicolumn{5}{|l|}{ Household factors } \\
\hline \multicolumn{5}{|l|}{ Household wealth quintile } \\
\hline 1 (Poorest) & 202 & $53(26.2)$ & 1.00 & 1.00 \\
\hline 2 & 197 & $71(36)$ & $1.30(0.94-1.80)$ & $1.25(0.9-1.73)$ \\
\hline 3 & 202 & $57(28.2)$ & $1.08(0.79-1.48)$ & $1.05(0.75-1.47)$ \\
\hline 4 & 205 & $65(31.7)$ & $1.20(0.88-1.65)$ & $1.07(0.78-1.46)$ \\
\hline 5 (Richest) & 200 & $83(41.5)$ & $1.59(1.13-2.25)^{*}$ & $1.45(0.98-2.14)$ \\
\hline \multicolumn{5}{|l|}{ Household head's education } \\
\hline None & 480 & $146(30.4)$ & 1.00 & 1.00 \\
\hline Some primary & 189 & $71(37.6)$ & $1.27(1.01,1.59)^{*}$ & $1.19(0.92-1.52)$ \\
\hline Secondary and above & 337 & $112(33.2)$ & $1.24(0.99-1.55)$ & $1.17(0.92-1.48)$ \\
\hline \multicolumn{5}{|l|}{ Cultivable land size (in $\mathrm{Ha}$ ) } \\
\hline Landless $(<0.1)$ & 421 & $121(28.7)$ & 1.00 & 1.00 \\
\hline Small size $(\geq 0.1 \&<0.5)$ & 259 & $81(31.3)$ & $1.20(0.98-1.47)$ & $1.18(0.97-1.43)$ \\
\hline Large size ( $\geq 0.5)$ & 326 & $127(39)$ & $1.26(1.00-1.58)$ & $1.21(0.96-1.52)$ \\
\hline
\end{tabular}


Table 4 Determinants of pre-lacteal feeding among infants in Nepal, 2013 a,b,c (Continued)

\begin{tabular}{|c|c|c|c|c|}
\hline Determinants & $n$ & Fed prelacteal feeds (\%) & $\begin{array}{l}\text { Model } 1 \text { (Unadjusted PR) } \\
\text { PR (95\% Cl) }\end{array}$ & $\begin{array}{l}\text { Model } 3^{\mathrm{d}} \text { (Adjusted PR) } \\
\text { APR }(95 \% \mathrm{Cl})\end{array}$ \\
\hline \multicolumn{5}{|c|}{ Contextual factors } \\
\hline \multicolumn{5}{|c|}{ Agro-ecological zones } \\
\hline Mountain & 160 & $23(14.4)$ & - & 1.00 \\
\hline Hill & 257 & $67(26.1)$ & & $1.49(0.83-2.65)$ \\
\hline Terai & 589 & $239(40.6)$ & & $\begin{array}{l}2.72 \\
(1.67-4.45)^{* *}\end{array}$ \\
\hline
\end{tabular}

${ }^{\mathrm{a}}$ For interpretation purposes, a PR $>1$ indicates that prelacteal feeding was more likely and PR $<1$ indicates that prelacteal feeding was less likely

$b_{*} P$-value $<0.05, * * P$-value $<0.001$

'(Model 2 shown in Additional file 3)

${ }^{\mathrm{d}}$ Model 3 included mother's education and visit by FCHVs for ANC as a priori covariates plus all variables that were significant $(p<0.2)$ in the first set of multivariable models

e"more highly trained healthcare providers" includes government health workers (MCHW/VHW, HA/AHW, Nurse/Midwife), doctors/pharmacists and NGO health workers

can still serve as an important breastfeeding indicator when information on exclusive breastfeeding is not available [32]. For an instance, a study done in Mexico showed that predominant breastfeeding is associated with lower gastrointestinal infection among infants at 6 months of age [43]. Another prospective cohort study done in Brazil showed that predominant breastfeeding increased the growth rate of infants in the first months of life [44].

Our findings suggest that introducing prelacteal feeds may disrupt the feeding of colostrum and increase the likelihood of other foods being introduced in the first 6 months, as has been reported in Ethiopia and other settings [45]. Prelacteal feeding has also been associated with delayed initiation of breastfeeding in Bangladesh [19]. In Nepal, prelacteal feeding has been associated with a higher risk of infant mortality in a dose-response manner [2], adding a compelling evidence for the need to breastfeed and avoid prelacteal feeds immediately after birth.

Our results reveal geographical differences in breastfeeding practices within Nepal. Feeding colostrum was less prevalent while the introduction of prelacteal feeds was more prevalent in the Terai. These observations are consistent with an earlier analysis suggesting that timely initiation of breastfeeding was $42 \%$ less likely in the Terai than mountains [46]. Ethnicity/caste was also associated with breastfeeding practices, with children from lower caste families being more likely to be predominantly breastfeed than upper caste families, possibly because the latter were more wealthy and able to afford breast milk substitutes. In the present study, mothers under 20 years of age were more likely to report timely initiation of breastfeeding, defined as breastfeeding within $1 \mathrm{~h}$ after birth, than older mothers, but less likely to report feeding their infants colostrum. Further research may might reveal reasons underlying differences in practice, including varied traditions among ethnic groups.

Infant age at assessment was an important predictor of predominant breastfeeding, with older children more likely to have already had semi-solid or solid foods introduced. This finding is consistent with findings from other studies in South Asia [47-49] and elsewhere [50-52] indicating a transition to complementary feeding in mid-infancy.

Maternal education did not appear to exert a strong influence on breastfeeding practices, unlike in Nepal [53], Bangladesh [54], India [55] and Pakistan [56] where women without formal education have been more likely to report a delay in the initiation of breastfeeding. An explanation may be irrespective of maternal education level and socioeconomic status if the mothers undergo caesarian section, they are less likely to initiate early breastfeeding [57]. Paradoxically, mothers having knowledge of the need to breastfeed through diarrheal episodes were less likely to predominantly breastfeed, possibly reflecting common occurrence of infantile diarrhea and an understood need to feed other fluids or foods during diarrhea.

Mothers engaged in agricultural occupations were more likely introduce breastfeeding shortly after birth, as seen elsewhere in Nepal [53]. In contrast, in households headed by salaried or agriculture workers, infants were less likely to receive colostrum. Reasons for different infant feeding patterns by occupation remain largely unknown and merit further exploration.

Households below the 20th percentile of our derived wealth index reported a higher prevalence of predominant breastfeeding than all wealthier groups, a finding that is consistent with studies from India [48] and Sri Lanka [58]. Possibly, wealthier women may be salaried workers, such as teachers, working in shops or self-employed (data not shown), thus finding it more difficulty to exclusively/predominantly breastfeed. On the other hand, households above the 80th percentile of the wealth index were more likely to feed colostrum to their newborns, consistent with practices observed in the District Level Household Survey (DLHS-3) of India where infants from richer households in non-Empowered 
Table 5 Determinants of predominant breastfeeding among children less than 6 months of age in Nepal, 2013 a,b,c

\begin{tabular}{|c|c|c|c|c|}
\hline Determinants & $n$ & Predominantly breastfed, $n(\%)$ & $\begin{array}{l}\text { Model } 1 \text { (Unadjusted PR) } \\
\text { PR ( } 95 \% \text { Cl) }\end{array}$ & $\begin{array}{l}\text { Model 3 } \\
\text { (Adjusted PR) } \\
\text { APR (95\% CI) }\end{array}$ \\
\hline Overall & 458 & $262(57.2)$ & & \\
\hline \multicolumn{5}{|l|}{ Child factors } \\
\hline \multicolumn{5}{|l|}{ Child's gender } \\
\hline Male & 247 & $141(57.1)$ & 1.00 & - \\
\hline Female & 211 & $121(57.4)$ & $1.01(0.86-1.18)$ & \\
\hline \multicolumn{5}{|l|}{ Age (in months) } \\
\hline 0 to 1.9 & 127 & $94(74.0)$ & 1.00 & 1.00 \\
\hline 2 to 3.9 & 171 & $108(63.2)$ & $0.84(0.73-0.98)^{*}$ & $0.86(0.75-0.98)^{*}$ \\
\hline 4 to 5.9 & 160 & $60(37.5)$ & $0.50(0.37-0.68)^{* *}$ & $0.57(0.42-0.77)^{* *}$ \\
\hline \multicolumn{5}{|l|}{ Child's birth order } \\
\hline First born child & 228 & $118(51.8)$ & 1.00 & - \\
\hline Second or later born child & 230 & $114(62.6)$ & $1.18(1.00-1.40)$ & \\
\hline \multicolumn{5}{|c|}{ Breastfed within one hour of birth } \\
\hline No & 251 & $130(51.8)$ & 1.00 & - \\
\hline Yes & 205 & $130(63.4)$ & $1.23(1.01,1.5)^{*}$ & \\
\hline \multicolumn{5}{|l|}{ Child fed colostrum } \\
\hline No & 73 & $43(58.9)$ & 1.00 & - \\
\hline Yes & 383 & $217(56.7)$ & $1(0.83,1.19)$ & \\
\hline \multicolumn{5}{|l|}{ Child fed prelacteal feeds } \\
\hline No & 305 & $214(70.2)$ & 1.00 & 1.00 \\
\hline Yes & 147 & $43(29.3)$ & $0.41(0.29,0.57)^{* *}$ & $0.45(0.32,0.62)^{* *}$ \\
\hline \multicolumn{5}{|l|}{ Maternal factors } \\
\hline \multicolumn{5}{|l|}{ Mother's education } \\
\hline None & 206 & $124(60.2)$ & 1.00 & 1.00 \\
\hline Some primary & 66 & $40(60.6)$ & $1.03(0.83-1.28)$ & $1.01(0.82-1.26)$ \\
\hline Secondary and above & 186 & $98(52.7)$ & $0.91(0.73-1.13)$ & $0.92(0.76-1.13)$ \\
\hline \multicolumn{5}{|l|}{ Visit by FCHV for ANC } \\
\hline No & 397 & $222(55.9)$ & 1.00 & 1.00 \\
\hline Yes & 61 & $40(65.6)$ & $1.14(0.91-1.42)$ & $1.11(0.90-1.38)$ \\
\hline \multicolumn{5}{|l|}{ Visit to health facilities for ANC } \\
\hline No & 127 & $65(51.2)$ & 1.00 & 1.00 \\
\hline Yes & 331 & $197(59.5)$ & $1.18(1.00-1.40)$ & $1.19(1.02-1.38)^{*}$ \\
\hline \multicolumn{5}{|l|}{ Visit by FCHV for postnatal care } \\
\hline No & 400 & $224(56)$ & 1.00 & - \\
\hline Yes & 58 & $38(65.5)$ & $1.26(0.98-1.61)$ & \\
\hline \multicolumn{5}{|c|}{ Visit to health facilities for postnatal care } \\
\hline No & 275 & $154(56.0)$ & 1.00 & 1.00 \\
\hline Yes & 183 & $108(59.0)$ & $1.12(0.96-1.32)$ & $1.00(0.87-1.15)$ \\
\hline \multicolumn{5}{|l|}{ Maternal knowledge present on } \\
\hline \multicolumn{5}{|c|}{ Exclusive breastfeeding for infants up to 6 months of age } \\
\hline No & 152 & $80(52.6)$ & 1.00 & 1.00 \\
\hline Yes & 306 & $182(59.5)$ & $1.19(1.03-1.39)^{*}$ & $1.19(1.01-1.39)^{*}$ \\
\hline
\end{tabular}

Breastfeeding for children during diarrhea 
Table 5 Determinants of predominant breastfeeding among children less than 6 months of age in Nepal, 2013 $3^{a, b, c}$ (Continued)

\begin{tabular}{|c|c|c|c|c|}
\hline Determinants & $n$ & Predominantly breastfed, $n(\%)$ & $\begin{array}{l}\text { Model } 1 \text { (Unadjusted PR) } \\
\text { PR (95\% CI) }\end{array}$ & $\begin{array}{l}\text { Model } 3^{\text {d }} \\
\text { (Adjusted PR) } \\
\text { APR ( } 95 \% \text { Cl) }\end{array}$ \\
\hline No & 370 & $222(60.0)$ & 1.00 & 1.00 \\
\hline Yes & 88 & $40(45.5)$ & $0.72(0.59-0.88)^{*}$ & $0.80(0.66-0.97)^{*}$ \\
\hline \multicolumn{5}{|c|}{ Women's empowerment (scale: $0-14, \mathrm{Md}=5$ ) } \\
\hline$<=8$ (less empowered) & 377 & $212(56.2)$ & 1.00 & \multirow[t]{2}{*}{-} \\
\hline$>=9$ (more empowered) & 81 & $50(61.7)$ & $1.15(0.93-1.42)$ & \\
\hline \multicolumn{5}{|l|}{ Household factors } \\
\hline \multicolumn{5}{|l|}{ Ethnicity/Caste } \\
\hline Upper caste & 111 & $55(49.6)$ & 1.00 & 1.00 \\
\hline Disadvantaged non-dalit Terai caste & 156 & $100(64.1)$ & $1.26(0.85-1.86)$ & $1.38(0.89-2.14)$ \\
\hline Janajatis & 101 & $44(43.6)$ & $0.95(0.66-1.36)$ & $1.02(0.75-1.39)$ \\
\hline Lower caste ${ }^{e}$ & 90 & $63(70.0)$ & $1.48(1.09-2.00)^{*}$ & $1.47(1.02-2.12)^{*}$ \\
\hline \multicolumn{5}{|l|}{ Household wealth quintile } \\
\hline 1 (Poorest) & 79 & $53(67.1)$ & 1.00 & 1.00 \\
\hline 2 & 91 & $47(51.7)$ & $0.66(0.49-0.91)^{*}$ & $0.68(0.51-0.91)^{*}$ \\
\hline 3 & 86 & $50(58.1)$ & $0.77(0.61-0.99)^{*}$ & $0.79(0.62-1.00)$ \\
\hline 4 & 100 & $61(61.0)$ & $0.82(0.64-1.05)$ & $0.87(0.7-1.07)$ \\
\hline 5 (Richest) & 102 & $51(50.0)$ & $0.71(0.49-1.04)$ & $0.79(0.58-1.08)$ \\
\hline \multicolumn{5}{|l|}{ Household head's education } \\
\hline None & 205 & $122(59.5)$ & 1.00 & 1.00 \\
\hline Some primary & 102 & $51(50)$ & $0.85(0.69-1.05)$ & $0.98(0.8-1.19)$ \\
\hline Secondary and above & 151 & $89(58.9)$ & $0.99(0.82-1.19)$ & $1.14(0.94-1.37)$ \\
\hline \multicolumn{5}{|l|}{ Community level factors } \\
\hline \multicolumn{5}{|l|}{ Agro-ecological zones } \\
\hline Mountain & 83 & $52(62.7)$ & - & 1.00 \\
\hline Hill & 116 & $47(40.5)$ & & $0.67(0.49-0.93)^{*}$ \\
\hline Terai & 259 & $163(62.9)$ & & $1.06(0.76-1.48)$ \\
\hline
\end{tabular}

${ }^{a}$ For interpretation purposes, a PR $>1$ indicates children were more likely to be predominantly breastfed and $\mathrm{PR}<1$ indicates children were less likely

$\mathrm{b}_{*} P$-value $<0.05,{ }^{* *} P$-value $<0.001$

${ }^{c}$ (Model 2 shown in Additional file 4)

${ }^{\mathrm{d}}$ Model 3 included mother's education and visit by FCHVs for ANC as a priori covariates plus all variables that were significant $(p<0.2)$ in the first set of multivariable models

e"Lower caste" includes Dalits and religious minorities

Action Group States were more likely than less wealthy homes to feed colostrum [59].

First born children were more likely to be fed prelacteal feeds than later siblings, consistent with observations from the 2011 NDHS [33]. In contrast, in Rupandehi District of Nepal, the odds of giving prelacteal feeds increased with parity [28], revealing possible variation in prelacteal feeding across Nepal, as has been observed with child feeding [60]. Surprisingly, mothers who reported receiving antenatal care from formally trained government health workers, doctors, pharmacists and NGO health workers were also more likely to give their infants prelacteal feeds, a pattern not observed with home visits from FCHVs. Visits to more highly trained providers may be have been due to maternal illness or obstetric emergencies (e.g., requiring caesarian section) making it difficult for mothers to initiate breastfeeding $[28,61]$. However, it has also been shown in Nepal that recommendations to mothers to use a breastmilk substitute from a health worker increases the likelihood of compliance with this practice than if no such guidance is given [62]. On the other hand, counseling during ANC about the importance of breastfeeding can influence a mother to initiate early breastfeeding [63]. Our findings provide support for continuing this approach in Nepal. Finally, the practice of feeding prelacteal feeds was more common in the Terai, consistent with observations from the NDHS [33], clearly identifying this 
region as one of high priority for intensified efforts to change this practice.

The main strengths of this study are that the sampling frame was designed to both statistically represent the country as well as the three major agro-ecological zones and that the survey content included a wide variety of potential determinants. Limitations of the study include its cross-sectional design and the reliance on predominant breastfeeding rather than exclusive breastfeeding as an outcome indicator, due to the lack of inclusion of plain water and other liquids in the 7-day recall. Additionally, we cannot rule out the possibility of social desirability bias or potential survival bias given the reliance on recall-based indicators and strong associations between breastfeeding and the risk of infant mortality.

\section{Conclusions}

Our study affirms a need to continue improving breastfeeding practices in rural Nepal through strengthened antenatal care and IYCF practices. Of particular concern is the need to reduce prelacteal feeding, especially in the southern plains (Terai) and encourage early introduction of breastfeeding, both of which may help extend the duration of predominant breastfeeding, and likely, exclusive breastfeeding. Increasing coverage of ANC check-ups and focusing efforts on early IYCF practices may be a useful way of improving coverage.

\section{Additional files}

Additional file 1: Determinants of breastfeeding within one hour of birth among infants in Nepal, 2013. This file contains model 2 in addition to other models presented in the main text. (PDF $153 \mathrm{~kb}$ )

Additional file 2: Determinants of feeding colostrum among infants in Nepal, 2013. This file contains model 2 in addition to other models presented in the main text. (PDF $183 \mathrm{~kb}$ )

Additional file 3: Determinants of prelacteal feeding among infants in Nepal, 2013. This file contains model 2 in addition to other models presented in the main text. (PDF $140 \mathrm{~kb}$ )

Additional file 4: Determinants of predominant breastfeeding among children less than 6 months of age in Nepal, 2013. This file contains model 2 in addition to other models presented in the main text (PDF $154 \mathrm{~kb})$

\section{Abbreviations}

AHW: Auxiliary Health Worker; ANC: Antenatal care; APR: Adjusted Prevalence Ratio; Cl: Confidence Interval; DHS: Demographic and Health Survey; FCHV: Female Community Health Volunteer; HA: Health Assistant; IYCF: Infant and Young Child Feeding; MCHW: Maternal and Child Health Worker; ORS: Oral Rehydration Solution; PHC: Primary Health Center; VDC: Village Development Committee; VHW: Village Health Worker; WHO: World Health Organization

\section{Acknowledgements}

The authors gratefully acknowledge New ERA as the data collection agency; the support of the Child Health Division, Department of Health Services, the Ministry of Health and Population of Nepal, and district offices of study sites; Ramesh K. Adhikari, Abhigyna Bhattarai, Devendra Gauchan, Dev Raj Gautam, Shibani Ghosh, Elena Broaddus, Dev Mandal Narayan, Ruchita Rajbhandary, Diplav Sapkota, Hari Krishna Shah and Patrick Webb; and thank the households and communities who participated in this study.

\section{Funding}

USAID Feed the Future Nutrition Innovation Lab, The US Agency for International Development (USAID), Washington DC, under the Cooperative Agreement AIDOAA-L-10-00005, with additional assistance from the Sight and Life Foundation and Bill \& Melinda Gates Foundation.

\section{Availability of data and materials}

The datasets used and/or analyzed during the current study are available from the corresponding author on reasonable request.

\section{Authors' contributions}

SB, ATL, RK, SM, KW designed the study. SB led data analysis. BS, SN, BASN and ATL assisted in data analysis. SB, ATL, SM, KW, BASN, BS and RK led data interpretation. SB and ATL led manuscript writing. SN and BS led data management. All authors have read and approved the final manuscript.

\section{Ethics approval and consent to participate}

The study was ethically approved by the by the Nepal Health Research Council (Reg. No.: 16/2013), an autonomous body, under the Ministry of Health, Government of Nepal, and the Institutional Review Board (IRB number: IRB00004937) at the Johns Hopkins Bloomberg School of Public Health, Baltimore, MD. Participants were briefed about the purpose of the study and participation was voluntary. If they agreed to be in the study, informed oral consent was obtained.

\section{Consent for publication}

Not applicable.

\section{Competing interests}

The authors declare that they have no competing interests.

\section{Publisher's Note}

Springer Nature remains neutral with regard to jurisdictional claims in published maps and institutional affiliations.

\section{Author details}

'PoSHAN Study Team, Lalitpur, Nepal. ${ }^{2}$ Department of Health Promotion, Education and Behavior, Arnold School of Public Health, University of South Carolina, Columbia, SC, USA. ${ }^{3}$ Center for Human Nutrition, Department of International Health, Johns Hopkins Bloomberg School of Public Health, Baltimore, MD, USA. ${ }^{4}$ Helen Keller International, New York, NY, USA.

Received: 7 October 2018 Accepted: 21 March 2019

Published online: 03 April 2019

References

1. WHO. Infant and Young Child feeding. Model chapter for textbooks for medical students and allied health professionals. Geneva: World Health Organization; 2009.

2. Lamichhane DK, Leem JH, Kim HC, Park MS, Lee JY, Moon SH, et al. Association of infant and young child feeding practices with undernutrition in children: evidence from the Nepal Demographic and Health Survey. Paediatr Int Child Health. 2016;36(4):260-9.

3. Mullany LC, Katz J, Li YM, Khatry SK, LeClerq SC, Darmstadt GL, et al. Breastfeeding patterns, time to initiation, and mortality risk among newborns in southern Nepal. J Nutr. 2008;138(3):599-603.

4. Garcia CR, Mullany LC, Rahmathullah L, Katz J, Thulasiraj RD, Sheeladevi S, et al. Breast-feeding initiation time and neonatal mortality risk among newborns in South India. J Perinatol. 2011;31(6):397-403.

5. Rochat TJ, Houle B, Stein A, Coovadia H, Coutsoudis A, Desmond C, et al. Exclusive breastfeeding and cognition, executive function, and behavioural disorders in primary school-aged children in rural South Africa: a cohort analysis. PLoS Med. 2016;13(6):e1002044.

6. Bar S, Milanaik R, Adesman A. Long-term neurodevelopmental benefits of breastfeeding. Curr Opin Pediatr. 2016;28(4):559-66.

7. Kamudoni P, Maleta K, Shi Z, Holmboe-Ottesen G. Exclusive breastfeeding duration during the first 6 months of life is positively associated with length-for-age among infants 6-12 months old, in Mangochi district, Malawi. Eur J Clin Nutr. 2015;69(1):96-101. 
8. Kuchenbecker J, Jordan I, Reinbott A, Herrmann J, Jeremias T, Kennedy G, et al. Exclusive breastfeeding and its effect on growth of Malawian infants: results from a cross-sectional study. Paediatr Int Child Health. 2015;35(1):14-23.

9. Horta BL, Loret de Mola C, Victora CG. Breastfeeding and intelligence: a systematic review and meta-analysis. Acta Paediatr. 2015;104(467):14-9.

10. World Health Organization. Exclusive breastfeeding for six months best for babies everywhere. 2011. http://www.who.int/mediacentre/news/ statements/2011/breastfeeding_20110115/en/. Accessed 4 Aug 2016.

11. McNeilly AS, Robinson IC, Houston MJ, Howie PW. Release of oxytocin and prolactin in response to suckling. Br Med J (Clin Res Ed). 1983;286(6361):257-9.

12. Ministry of Health, New ERA, ICF International Inc. Demographic and Health Survey 2016: key indicators. Kathmandu: Minisetry of Health; 2017.

13. Ministry of Health, New ERA, ICF International Inc. Nepal Demographic and Health Survey 2016. Kathmandu: Ministry of Health; 2017.

14. Thapa M. Traditional beliefs and practices in newborn care in Nepal. In: Costello A, Manandhar D, editors. Improving newborn infant health in developing countries. London: Imperial College Press; 2000. p. 181-7.

15. Bandyopadhyay M. Impact of ritual pollution on lactation and breastfeeding practices in rural West Bengal, India. Int Breastfeed J. 2009;4:2.

16. Laroia N, Sharma D. The religious and cultural bases for breastfeeding practices among the Hindus. Breastfeed Med. 2006;1(2):94-8.

17. McKenna KM, Shankar RT. The practice of prelacteal feeding to newborns among Hindu and Muslim families. J Midwifery Womens Health. 2009;54(1):78-81.

18. Raina SK, Mengi V, Singh G. Differentials in colostrum feeding among lactating women of block RS Pura of $\mathrm{J}$ and $\mathrm{K}$ : a lesson for nursing practice. Iran J Nurs Midwifery Res. 2012;17(5):386-9.

19. Sundaram ME, Ali H, Mehra S, Shamim AA, Ullah B, Rashid M, et al. Early newborn ritual foods correlate with delayed breastfeeding initiation in rural Bangladesh. Int Breastfeed J. 2016;11:31.

20. Bililign N, Kumsa H, Mulugeta M, Sisay Y. Factors associated with prelacteal feeding in north eastern Ethiopia: a community based cross-sectional study. Int Breastfeed J. 2016;11:13.

21. Legesse M, Demena M, Mesfin F, Haile D. Factors associated with colostrum avoidance among mothers of children aged less than 24 months in Raya kobo district, North-Eastern Ethiopia: community-based cross-sectional study. J Trop Pediatr. 2015;61(5):357-63.

22. Cacho NT, Lawrence RM. Innate immunity and breast milk. Front Immunol. 2017:8:584.

23. Goldman AS. Modulation of the gastrointestinal tract of infants by human milk. Interfaces and interactions. An evolutionary perspective. J Nutr. 2000; 130(2S Suppl):426S-31S.

24. Hajeebhoy N, Nguyen PH, Mannava P, Nguyen TT, Mai LT. Suboptimal breastfeeding practices are associated with infant illness in Vietnam. Int Breastfeed J. 2014;9:12.

25. Thow AM, Karn S, Devkota MD, Rasheed S, Roy S, Suleman Y, et al. Opportunities for strengthening infant and young child feeding policies in South Asia: insights from the SAIFRN policy analysis project. BMC Public Health. 2017;17(2):404.

26. Development Initiatives. Nutrition country profile, Nepal. 2018. https:// globalnutritionreport.org/nutrition-profiles/asia/southern-asia/nepal/. Accessed 05 Jan 2019.

27. Karkee $\mathrm{R}$, Lee $\mathrm{AH}$, Khanal $\mathrm{V}$, Binns CW. Infant feeding information, attitudes and practices: a longitudinal survey in Central Nepal. Int Breastfeed J. 2014;9:14.

28. Khanal V, Lee AH, Karkee R, Binns CW. Prevalence and factors associated with prelacteal feeding in Western Nepal. Women Birth. 2016;29(1):12-7.

29. Ulak M, Chandyo RK, Mellander L, Shrestha PS, Strand TA. Infant feeding practices in Bhaktapur, Nepal: a cross-sectional, health facility based survey Int Breastfeed J. 2012;7:1.

30. Klemm R, Manohar S, Rajbhandary R, Shrestha K, Gauchan D, Adhikari R, et al. Pathways from agriculture-to-nutrition: design and conduct of the national PoSHAN surveys of Nepal. J Food Security. 2018;6(2):79-89.

31. Launer LJ, Forman MR, Hundt GL, Sarov B, Chang D, Berendes HW, et al. Maternal recall of infant feeding events is accurate. J Epidemiol Community Health. 1992;46(3):203-6.

32. World Health Organization. Indicators for assessing infant and young child feeding practices: conclusions of a consensus meeting held 6-8 November 2007 in Washington D.C., USA. Geneva: WHO; 2008.

33. Khanal V, Adhikari M, Sauer K, Zhao Y. Factors associated with the introduction of prelacteal feeds in Nepal: findings from the Nepal demographic and health survey 2011. Int Breastfeed J. 2013;8:9.
34. Khanal V, Sauer K, Zhao Y. Exclusive breastfeeding practices in relation to social and health determinants: a comparison of the 2006 and 2011 Nepa demographic and health surveys. BMC Public Health. 2013;13:958.

35. Rutstein SO, Johnson K. The DHS wealth index. Calverton, Maryland: ORC Macro; 2004.

36. Thompson ML, Myers JE, Kriebel D. Prevalence odds ratio or prevalence ratio in the analysis of cross sectional data: what is to be done? Occup Environ Med. 1998:55(4):272-7.

37. Alexander LK, Lopes B, Ricchetti-Masterson K, Yeatts KB. ERIC note book: cross-sectional studies. https://sph.unc.edu/files/2015/07/nciph_ERIC8.pdf. Accessed 2 Nov 2017

38. Lindquist K. How can I estimate relative risk using GLM for common outcomes in cohort studies? 2017. http://stats.idre.ucla.edu/stata/faq/howcan-i-estimate-relative-risk-using-glm-for-common-outcomes-in-cohortstudies/. Accessed 16 Nov 2017.

39. Zou G. A modified Poisson regression approach to prospective studies with binary data. Am J Epidemiol. 2004;159(7):702-6.

40. Acharya $P$, Khanal $V$. The effect of mother's educational status on early initiation of breastfeeding: further analysis of three consecutive Nepal demographic and health surveys. BMC Public Health. 2015;15:1069.

41. Khatri RB, Mishra SR, Khanal V. Female community health volunteers in community-based health programs of Nepal: future perspective. Front Public Health. 2017:5:181.

42. Fenta EH, Yirgu R, Shikur B, Gebreyesus SH. A single 24 h recall overestimates exclusive breastfeeding practices among infants aged less than six months in rural Ethiopia. Int Breastfeed J. 2017;12:36.

43. Monterrosa EC, Frongillo EA, Vásquez-Garibay EM, Romero-Velarde E, Casey LM, Willows ND. Predominant breast-feeding from birth to six months is associated with fewer gastrointestinal infections and increased risk for iron deficiency among infants. J Nutr. 2008;138(8):1499-504.

44. Spyrides MHC, Struchiner CJ, Barbosa MTS, Kac G. Effect of predominant breastfeeding duration on infant growth: a prospective study using nonlinear mixed effect models. J Pediatr. 2008;84:237-43.

45. Tewabe T, Mandesh A, Gualu T, Alem G, Mekuria G, Zeleke H. Exclusive breastfeeding practice and associated factors among mothers in Motta town, east Gojjam zone, Amhara regional state, Ethiopia, 2015: a crosssectional study. Int Breastfeed J. 2017;12:12.

46. Pandey JP, Dhakal MR, Karki S, Poudel P, Pradhan MS. Maternal and child health in Nepal: the effects of caste, ethnicity, and regional identity: further analysis of the 2011 Nepal Demographic and Health Survey. Calverton, MD: Nepal Ministry of Health and Population, New ERA, and ICF International; 2013.

47. Perera PJ, Ranathunga N, Fernando MP, Sampath W, Samaranayake GB. Actual exclusive breastfeeding rates and determinants among a cohort of children living in Gampaha district Sri Lanka: a prospective observational study. Int Breastfeed J. 2012;7:21.

48. Chandhiok N, Singh K, Sahu D, Singh L, Pandey A. Changes in exclusive breastfeeding practices and its determinants in India, 1992-2006: analysis of national survey data. Int Breastfeed J. 2015;10:34

49. Akter S, Rahman MM. Duration of breastfeeding and its correlates in Bangladesh. J Health Popul Nutr. 2010;28(6):595-601.

50. Thu HN, Eriksson B, Khanh TT, Petzold M, Bondjers G, Kim CNT, et al. Breastfeeding practices in urban and rural Vietnam. BMC Public Health. 2012;12:964.

51. Onah S, Osuorah DIC, Ebenebe J, Ezechukwu C, Ekwochi U, Ndukwu I. Infant feeding practices and maternal socio-demographic factors that influence practice of exclusive breastfeeding among mothers in Nnewi south-East Nigeria: a cross-sectional and analytical study. Int Breastfeed J. 2014;9:6.

52. Victor R, Baines SK, Agho KE, Dibley MJ. Determinants of breastfeeding indicators among children less than 24 months of age in Tanzania: a secondary analysis of the 2010 Tanzania demographic and health survey. BMJ Open. 2013;3:e001529.

53. Adhikari M, Khanal V, Karkee R, Gavidia T. Factors associated with early initiation of breastfeeding among Nepalese mothers: further analysis of Nepal demographic and health survey, 2011. Int Breastfeed J. 2014;9:21.

54. Mihrshahi S, Kabir I, Roy SK, Agho KE, Senarath U, Dibley MJ. Determinants of infant and young child feeding practices in Bangladesh: secondary data analysis of demographic and health survey 2004. Food Nutr Bull. 2010;31(2):295-313.

55. Patel A, Badhoniya N, Khadse S, Senarath U, Agho KE, Dibley MJ. Infant and young child feeding indicators and determinants of poor feeding practices in India: secondary data analysis of National Family Health Survey 2005-06. Food Nutr Bull. 2010;31(2):314-33. 
56. Ali S, Ali SF, Imam AM, Ayub S, Billoo AG. Perception and practices of breastfeeding of infants 0-6 months in an urban and a semi-urban community in Pakistan: a cross-sectional study. J Pak Med Assoc. 2011; 61(1):99-104

57. Khanal V, Scott JA, Lee AH, Karkee R, Binns CW. Factors associated with early initiation of breastfeeding in Western Nepal. Int J Environ Res Public Health. 2015;12(8):9562-74.

58. Senarath U, Siriwardena I, Godakandage SS, Jayawickrama H, Fernando DN, Dibley MJ. Determinants of breastfeeding practices: an analysis of the Sri Lanka demographic and health survey 2006-2007. Matern Child Nutr. 2012; 8(3):315-29.

59. Kumar A, Unisa S, Sharma B. Infant and young child feeding practices in India: a comparison of empowered action group (EAG) and non-EAG states. Soc Sci Spectr. 2017;3(1):52-64.

60. Broaddus-Shea ET, Thorne-Lyman AL, Manohar S, Nonyane BAS, Winch PJ, West KP Jr. Seasonality of consumption of nonstaple nutritious foods among young children from Nepal's 3 agroecological zones. Curr Dev Nutr. 2018;2(9):nzy058

61. Karkee R, Lee AH, Khanal V, Binns CW. Initiation of breastfeeding and factors associated with prelacteal feeds in Central Nepal. J Hum Lact. 2014;30(3):353-7.

62. Pries AM, Huffman SL, Adhikary I, Upreti SR, Dhungel S, Champeny M, et al. Promotion and prelacteal feeding of breastmilk substitutes among mothers in Kathmandu Valley, Nepal. Matern Child Nutr. 2016;12(Suppl 2):8-21.

63. Dhandapany G, Bethou A, Arunagirinathan A, Ananthakrishnan S. Antenatal counseling on breastfeeding - is it adequate? A descriptive study from Pondicherry, India. Int Breastfeed J. 2008;3:5.

Ready to submit your research? Choose BMC and benefit from:

- fast, convenient online submission

- thorough peer review by experienced researchers in your field

- rapid publication on acceptance

- support for research data, including large and complex data types

- gold Open Access which fosters wider collaboration and increased citations

- maximum visibility for your research: over $100 \mathrm{M}$ website views per year

At BMC, research is always in progress.

Learn more biomedcentral.com/submissions 